\title{
Suspected Wolf-Rayet Galaxies UM 456 and UM 594
}

\author{
A. Niedzielski and K. Rochowicz \\ Toruń Center for Astronomy (TCfA), N. Copernicus University, ul. Gagarina 11, 87-100 Toruń, Poland \\ Received 16 January 2002 / Accepted 8 april 2002

\begin{abstract}
First results of an on-going search for Wolf-Rayet galaxies, based on new observations obtained with the Hobby Eberly Telescope ${ }^{\star}$ (HET) and the Marcario Low Resolution Spectrograph ${ }^{\star \star}$ (LORES), are presented. UM 456 and UM 594 have been found to be normal HII galaxies. New determinations of radial velocities have been given: UM $456-V_{\mathrm{r}}=1702 \pm 48 \mathrm{~km} \mathrm{~s}^{-1} \mathrm{UM} 594-V_{\mathrm{r}}=6540 \pm 45 \mathrm{~km} \mathrm{~s}^{-1}$. Independent estimates of distances, $\mathrm{WR} /(\mathrm{WR}+\mathrm{O})$ ratios and oxygen to helium abundances ratios have been obtained.
\end{abstract}

Key words. galaxies: starburst - galaxies: stellar content - galaxies: individual: UM 456, UM $594-$ stars: Wolf-Rayet - stars: early-type

\section{Introduction}

The Wolf-Rayet populations in the Local Group of Galaxies are well resolved with contemporary telescopes. These populations are however very scarce as compared to those in other active star-creating galaxies - starbursts. Since the most massive stars evolve in extremely short time, already several hundred thousand years after a starburst we observe Wolf-Rayet stars, evolutionary advanced objects, just before the supernova explosion. Most intensive starbursts lead to formation of thousands of Wolf-Rayet stars and we can observe their characteristic spectral features in the integrated light of starburst host galaxies. We call such galaxies Wolf-Rayet galaxies. Systematic studies of Wolf-Rayet galaxies lead to important improvement of our knowledge on stellar populations and evolution as well as on the nature and evolution of galaxies.

From the number of Wolf-Rayet stars present in a starburst one can learn about the time-scale and intensity of the starburst phenomenon (the star formation rate), and about the initial mass function, its slope and especially its extent to high masses. The number of Wolf-Rayet stars

Send offprint requests to: A. Niedzielski,

e-mail: aniedzi@astri.uni.torun.pl

* The Hobby-Eberly Telescope is operated by McDonald Observatory on behalf of The University of Texas at Austin, the Pennsylvania State University, Stanford University, Ludwig-Maximilians-Universitaet München, and GeorgAugust-Universiteat Göttingen.

$\star \star$ The Marcario Low Resolution Spectrograph is a joint project of the Hobby-Eberly Telescope partnership and the Instituto de Astronomia de la Universidad Nacional Autonoma de Mexico. present in a starburst allows also to address other ingredients required for stellar population synthesis models like the intensity of stellar winds and metallicity.

Wolf-Rayet stars exhibit very characteristic emissionline spectra that, if their population is numerous enough, can be detected in the spectra of galaxies. Most prominent are emission lines of HeII $\lambda 1640 \AA$ in the UV and the so-called "WR bump" at $\lambda \sim 4650 \AA$ in the optical. The WR bump is composed of several emission lines of nitrogen (N III/N IV $\lambda 4640 \AA)$ carbon (C III/C IV $\lambda 4650 \AA)$ and helium (He II $\lambda 4686 \AA$ ). The intensity of individual lines varies with the WR spectral subtype. At lower resolutions, where individual components are not resolved, the WR bump is observed as a single, relatively wide feature.

Wolf-Rayet galaxies (WRG) were first pointed out by Allen et al. (1976) and catalogued (37 objects) by Conti (1991). Optical spectra of WRG are described in Vacca \& Conti (1992). The latest catalog of WRG (Schaerer et al. 1999) contains as many as 139 known and 48 suspected WR galaxies.

\section{Observations and reduction}

The spectra presented below were obtained with the Hobby Eberly Telescope (HET) Low Resolution Spectrograph (LORES) on March 14 and 15, 2000. LORES was set up with the second grism $(600 \mathrm{l} / \mathrm{mm}$, $4300-7300 \AA)$, and the slit was set to $2 \operatorname{arcsec}(R=630$ at $5950 \AA)$. In addition the default GG385 blocking filter was used. The spectra were recorded with a CCD camera with the Ford Aerospace CCD 3072x1024 with $15 \mu \mathrm{m}$ pixels binned $2 \times 2$. The resulting image scale is $0.235 \mathrm{arcsec} / \mathrm{pixel}$. Along with object spectra, a number of bias and flat-field frames, as well as calibration spectra of 
Table 1. Journal of observations.

\begin{tabular}{lllll}
\hline \hline Object & PA & Date & UT & Exp.[s] \\
\hline UM 594 & $90 \mathrm{E}$ & Mar. 14, 2000 & $10: 26: 57.05$ & 300 \\
UM 594 & $90 \mathrm{E}$ & Mar. 14, 2000 & $10: 34: 01.43$ & 300 \\
UM 456 & $23 \mathrm{E}$ & Mar. 15, 2000 & $08: 46: 54.83$ & 300 \\
\hline
\end{tabular}

Table 2. The list of lines identified in the optical spectrum of UM 456.

\begin{tabular}{lllll}
\hline \hline$\lambda_{\text {obs }}$ & ident. & $I_{\mathrm{c}}$ & $E W$ & $\lambda_{\text {lab }}$ \\
\hline 4352.89 & $\mathrm{H}_{\gamma} ?$ & 2.3 & -10.8 & 4340.46 \\
4885.53 & $\mathrm{H}_{\beta} 4861$ & 3.6 & -34.1 & 4861.32 \\
4984.57 & {$[\mathrm{OIII}] 4959$} & 5.4 & -58.7 & 4958.9 \\
5033.19 & {$[\mathrm{OIII}] 5007$} & 16.2 & -153.2 & 5006.9 \\
5907.81 & $\mathrm{HeI} 5876$ & 0.9 & -4.8 & 5875.6 \\
6335.20 & {$[\mathrm{OI}] 6300$} & 0.6 & -5.2 & 6302.03 \\
6581.64 & {$[\mathrm{NII}] 6548$} & 0.67 & -1.6 & 6549.86 \\
6599.30 & $\mathrm{H}{ }_{\alpha} 6562$ & 20.2 & -202 & 6562.8 \\
6621.17 & {$[\mathrm{NII}] 6584$} & 1.0 & -7.8 & 6585.27 \\
6753.90 & {$[\mathrm{SII}] 6717$} & 1.8 & -19.2 & 6718.26 \\
6768.90 & {$[\mathrm{SII}] 6731$} & 1.5 & -14.0 & 6732.65 \\
\hline
\end{tabular}

$\mathrm{HgCdZn}$ and Ne lamps were recorded. The spectra were obtained by a resident astronomer in the service mode. The CCD reduction was performed with IRAF standard packages in TCfA. The resulting spectra were obtained in 4250-7400 $\AA$ range, with the constant step of $2 \AA /$ pix. The resolution, determined by measuring FWHM of calibration lamp lines, was found to agree with the values given in the LORES manual. The journal of observations is presented in Table 1.

\section{Results}

\subsection{UM 456}

UM 456 (PGC 37019, SCHG 1148+002) is a $15.4(V)$ magnitude galaxy, whose spectrum was classified by Terlevich et al. (1991) as HII. It was placed on the list of suspected WRG by Schaerer et al. (1999). Our optical spectrum of this object is presented in Fig. 1. We obtained the $S / N$ ratio of about 10 at $5200 \AA$ and $5700 \AA$ (continuum). In Table 2 we present the measurements for the most prominent lines identified in the resulting spectrum: observed wavelengths, line central intensities (in continuum units) and equivalent widths (in $\AA$ ). The achieved $S / N$ ratio enabled us to identify lines stronger than $\sim 0.5 \AA(E W)$.

Using the classification criteria of Veilleux \& Osterbrock (1987), namely the $\log [\mathrm{OIII}]_{\lambda} 5007 / \mathrm{H}_{\beta}$ vs. $\log [\mathrm{SII}]_{\lambda} 6717+6731 / \mathrm{H}_{\alpha}, \log [\mathrm{OIII}]_{\lambda} 5007 / \mathrm{H}_{\beta}$ vs. $\log [\mathrm{OI}]_{\lambda}$ $6300 / \mathrm{H}_{\alpha}$ and $\log [\mathrm{OIII}]_{\lambda} 5007 / \mathrm{H}_{\beta}$ vs. $\log [\mathrm{NII}]_{\lambda} 6584 / \mathrm{H}_{\alpha}$ diagrams, and our measurements listed in Table 2 we confirm the spectral classification of Terlevich et al. (1991)

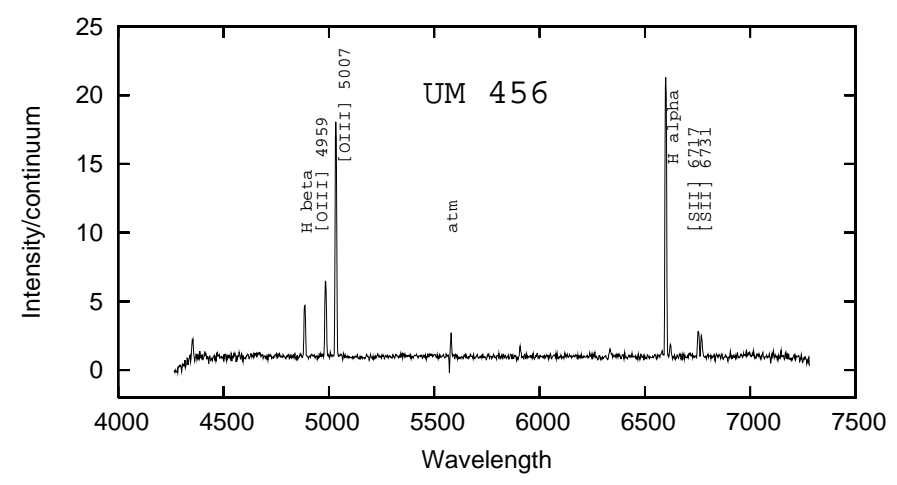

Fig. 1. The observed spectrum of UM 456. Wavelength is given in $\AA$.

and classify UM 456 as a normal HII galaxy. We have found no WR features in the presented optical spectrum of UM 456.

Following Schaerer \& Vacca (1998) we estimated the number of WR stars relative to the total population of WR and $\mathrm{O}$ stars from intensities of the WR bump and the $\mathrm{H}_{\beta}$. According to their Eq. (17) and assuming that the WR bump in UM 456 is as weak as the weakest lines observable in our spectrum we have estimated the upper limit of $\mathrm{WR} /(\mathrm{WR}+\mathrm{O})=0.02$. This ratio is very low, similar to the galactic one. The $\mathrm{WR} /(\mathrm{WR}+\mathrm{O})$ ratio for the Galaxy ranges between 0.02 and 0.10 , depending on estimates of the total numbers of $\mathrm{O}$ and WR stars. Assuming $N_{\mathrm{WR}}^{\text {total }}=$ 6500 according to van der Hucht $(2001)$, and $N_{\mathrm{O}-\mathrm{B} 2}^{\text {total }}=$ 60000 from Reed (2000), we obtain $\mathrm{WR} /(\mathrm{WR}+\mathrm{O})=0.10$.

We have determined the radial velocity (redshift) of UM 456 at $1702 \pm 48 \mathrm{~km} \mathrm{~s}^{-1}$ (reduced to the GSR). It was also determined by Grogin et al. (1998), who found $1756 \pm 70 \mathrm{~km} \mathrm{~s}^{-1}$. Our determination is more precise and the obtained value is smaller, but both determinations agree within the observational errors. Using our value of the radial velocity we estimated the distance to UM 456. Assuming $H_{0}=65 \pm 6 \mathrm{~km} \mathrm{~s}^{-1} \mathrm{Mpc}^{-1}$ (Hendry et al. 2001) we have obtained $26 \pm 3 \mathrm{Mpc}$.

From the data presented in Table 2 we estimated the $\mathrm{O} / \mathrm{H}$ ratio with the semi-empirical relation of RobledoRella \& Conti (1993). In the case of UM 456 the oxygen to helium ratio relative to the solar value $\left((\mathrm{O} / \mathrm{H})_{\odot}=6.76 \times\right.$ $10^{-4}$ - Grevesse \& Sauval 1998) is $\mathrm{O} / \mathrm{H}=0.18$, which suggests that it is metal deficient.

\subsection{UM 594}

UM 594 (SCHG $1335+002$, z17-43) is a $15.6(\mathrm{~V})$ magnitude galaxy classified by Terlevich et al. (1991) as "HII?" with the blue continuum visible. This galaxy was placed by Schaerer et al. (1999) on their list of suspected WRG. Its optical spectrum obtained by us is presented in Fig. 2. We reached the $S / N$ ratio of about 25 at $5300 \AA$ and $5700 \AA$ (continuum). Table 3 contains identification of the strongest emission lines, observed wavelengths, line central intensities (in continuum units) and equivalent 


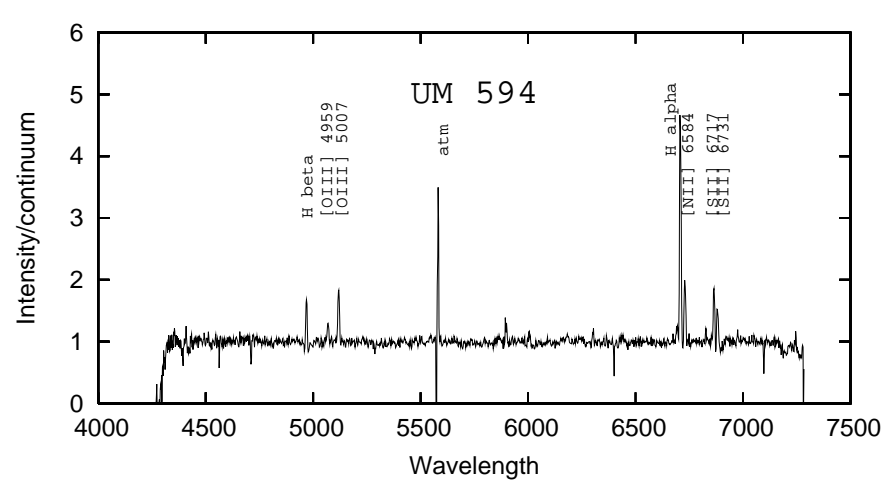

Fig. 2. The observed spectrum of UM 594. Wavelength is given in $\AA$.

Table 3. The list of lines identified in the optical spectrum of UM 594.

\begin{tabular}{|c|c|c|c|c|}
\hline$\lambda_{\mathrm{obs}}$ & ident. & $I_{\mathrm{c}}$ & $E W$ & $\lambda_{\text {lab }}$ \\
\hline 4419.92 & $\mathrm{H}_{\gamma} ?$ & -0.15 & 1.7 & 4340.46 \\
\hline 4562.82 & HeI $4471 ?$ & -0.6 & 1.0 & 4471.5 \\
\hline 4968.68 & $\mathrm{H}_{\beta} 4861+\mathrm{abs}$ & 0.9 & 5.9 & 4861.32 \\
\hline 5068.86 & [OIII] 4959 & 0.3 & -2.9 & 4958.9 \\
\hline 5118.48 & [OIII] 5007 & 0.9 & -8.3 & 5006.9 \\
\hline 6004.81 & HeI 5876 & 0.2 & -4.2 & 5876.6 \\
\hline 6439.56 & [OI] 6300? & 0.07 & -1.5 & 6302.03 \\
\hline 6692.39 & [NII] 6548 & 0.24 & -2.7 & 6549.86 \\
\hline 6708.30 & $\mathrm{H}_{\alpha} 6561$ & 3.85 & -34.7 & 6562.80 \\
\hline 6729.70 & [NII] 6584 & 1.04 & -10.0 & 6585.27 \\
\hline 6827.61 & HeI 6678 & 0.25 & -1.2 & 6678 \\
\hline 6864.96 & [SII] 6717 & 0.92 & -7.1 & 6718.26 \\
\hline 6881.61 & [SII] $6731+\mathrm{abs}$ & 0.56 & -4.7 & 6732.65 \\
\hline
\end{tabular}

widths $(E W)$. With the obtained $S / N$ ratio we were able to identify lines stronger than $E W \sim 0.25 \AA$.

Using the classification criteria of Veilleux \& Osterbrock (1987) and our measurements presented in Table 3 we classified UM 594 as a normal HII galaxy. We have found no WR features in the obtained optical spectrum of UM 594.

Using the same method as in the case of UM 456 we have estimated the upper limit of the $\mathrm{WR} /(\mathrm{WR}+\mathrm{O})$ ratio $=0.05$, again comparable to the galactic value.

The mean value of the radial velocity is $6540 \pm 45 \mathrm{kms}^{-1}$ (relative to the GSR). The redshift to UM 594 was also quite recently obtained by Terlevich et al. (1991). They got $z=+0.02$. Probably due to better quality of our spectra the radial velocity determined in the present paper is much more precise and almost $10 \%$ higher. Our determination agrees better with the value of $6653 \mathrm{~km} \mathrm{~s}^{-1}$ listed in Schaerer et al. (1999). Using our determination of the radial velocity and $H_{0}=$ $65 \pm 6 \mathrm{~km} \mathrm{~s}^{-1} \mathrm{Mpc}^{-1}$ (Hendry et al. 2001) we can estimate distance to UM 594 at $101 \pm 10 \mathrm{Mpc}$.

By applying the semi-empirical relation of RobledoRella \& Conti (1993) for UM 594 we estimated the oxygen to helium ratio relative to the solar value. We have found $\mathrm{O} / \mathrm{H}=0.66$. This value suggests that UM 594 is also metal deficient.

\section{Conclusions}

Basing on low resolution spectra obtained with the Marcario LORES spectrograph mounted in the prime focus of the Hobby-Eberly telescope two of suspected WR galaxies from the list of Schaerer et al. (1999), UM 456 and UM 594, have been found to be normal HII galaxies without any WR features. The strongest lines in the observed spectra of both galaxies have been identified and their equivalent widths and central intensities measured. The radial velocities, distances, upper limits of the WR star contents, as well as estimates of the oxygen abundances for both galaxies have been determined.

Acknowledgements. Kind cooperation of Prof. A. Wolszczan in this project is acknowledged with thanks.

\section{References}

Allen, D. A., Wright, A. E., \& Goss, W. M. 1976, MNRAS, 177, 91

Conti, P. S. 1991, ApJ, 377, 115

Grevesse, N., \& Sauval, A. J. 1998, Space Sci. Rev., 85, 161

Grogin, N. A., Geller, M. J., \& Huchara, J. P. 1998, ApJS, 119, 277

Hendry, M. A., Rauzy, S., Goodwin, S. P., \& Gribbin, J. 2001, MNRAS, 324, 717

van der Hucht, K. A. 2001, New Astr. Rev., 45, 135

Reed, B. C. 2000, AJ, 120, 314

Robledo-Rella, V., \& Conti, P. S. 1993, in Massive Stars: Their Lives in the Interstellar Medium, ASP Conf. Ser., 35, ed. J. P. Cassinelli, \& E. B. Churchwell, 534

Schaerer, D., Contini, T., \& Pindao, M. 1999, A\&ASS, 136, 35

Schaerer, D., \& Vacca, W. D. 1998, ApJ, 497, 618

Terlevich, R., Melnick, J., Masegosa, J., Moles, M., \& Copetti, M. V. F. 1991, A\&ASS, 91, 285

Vacca, W. D., \& Conti, P. S. 1992, ApJ, 401, 543

Veilleux, S., \& Osterbrock, D. E. 1987, ApJS, 63, 295 